\title{
Coherence and Polarization of Polarization Speckle Generated by Depolarizers and Their Changes through Complex ABCD Matrix
}

\author{
Ma, Ning; Hanson, Steen Grüner; Lee, Tim K.; Takeda, Mitsuo; Wang, Wei
}

\section{Published in:}

Proceedings of SPIE

Link to article, DOI:

$10.1117 / 12.2189940$

Publication date:

2015

Document Version

Publisher's PDF, also known as Version of record

Link back to DTU Orbit

Citation (APA):

Ma, N., Hanson, S. G., Lee, T. K., Takeda, M., \& Wang, W. (2015). Coherence and Polarization of Polarization Speckle Generated by Depolarizers and Their Changes through Complex ABCD Matrix. In Proceedings of SPIE (Vol. 9660). [96601D] SPIE - International Society for Optical Engineering. Proceedings of SPIE - The International Society for Optical Engineering https://doi.org/10.1117/12.2189940

\section{General rights}

Copyright and moral rights for the publications made accessible in the public portal are retained by the authors and/or other copyright owners and it is a condition of accessing publications that users recognise and abide by the legal requirements associated with these rights.

- Users may download and print one copy of any publication from the public portal for the purpose of private study or research.

- You may not further distribute the material or use it for any profit-making activity or commercial gain

- You may freely distribute the URL identifying the publication in the public portal 


\title{
Coherence and Polarization of Polarization Speckle Generated by Depolarizers and Their Changes through Complex ABCD Matrix
}

\author{
Ning Ma*a, Steen G. Hanson ${ }^{b}$, Tim K. Lee ${ }^{c}$, Mitsuo Takeda ${ }^{d}$, Wei Wang ${ }^{a}$ \\ ${ }^{a}$ Institute of Photonics \& Quantum Sciences, Heriot-Watt University, Edinburgh, UK \\ ${ }^{\mathrm{b}}$ DTU Fotonik, Technical University of Denmark, Roskilde, Denmark \\ ${ }^{c}$ Cancer Control Research Program, BC Cancer Agency, Vancouver, Canada \\ ${ }^{\mathrm{d} C e n t e r}$ for Optical Research and Education, Utsunomiya University, Utsunomiya, Japan
}

\begin{abstract}
Recent research work on speckle patterns indicates a variation of the polarization state during propagation and its nonuniformly spatial distribution. The preliminary step for the investigation of this polarization speckle is the generation of the corresponding field. In this paper, a kind of special depolarizer: the random roughness birefringent screen (RRBS) is introduced to meet this requirement. The statistical properties of the field generated by the depolarizer is investigated and illustrated in terms of the $2 \times 2$ beam coherence and polarization matrix (BCPM) with the corresponding degree of coherence (DoC) $\eta$ and degree of polarization (DoP) P. The changes of the coherence and polarization when the speckle field propagates through any optical system are analysed within the framework of the complex ABCD-matrix theory.
\end{abstract}

Keywords: statistical optics, laser speckle, coherence, polarization, depolarizer, ABCD matrix

\section{INTRODUCTION}

The speckle pattern is one of the most important concepts in optics which describes the phenomena of fine-scale, highcontrast granular light intensity distribution caused by the interference of a coherent optical field within the scattering spot $^{1}$. In the majority of studies on speckle phenomena, the random field is usually treated as a scalar random optical field. The main interest was in the statistical properties of their intensity and its changes during propagation, and it is implicitly asserted that polarization state will be maintained. Recent research work, both theoretical and experimental, indicates the variation of the polarization state during propagation and its non-uniform spatial distribution for the cross section of a speckle ${ }^{2-6}$. This special kind of speckle is named polarization speckle ${ }^{6}$ or electromagnetic beam ${ }^{4}$. To illustrate the polarization variation together with the coherence property of fields, a $2 \times 2$ beam coherence and polarization matrix (BCPM) in the spatial-temporal domain was developed by F. Gori ${ }^{7}$ and then expanded by E. Wolf to establish a unified theory of coherence and polarization within this framework ${ }^{8}$.

The basic premise of the investigation for the statistical properties of polarization speckle is the special kind of device/system which does not only diffuse the incident field but also scrambles its polarization like a depolarizer. Liquid-crystal (LC) spatial light modulators (SLMs) modulating only one of the two orthogonal field components were introduced as one of the most promising anisotropic depolarizer to give rise to a prescribed speckle field with particular spatial polarization properties ${ }^{9}$. The modulated field associated with the propagation in free space were investigated statistically, in terms of the BCPM implicating the polarization and coherence properties ${ }^{4}$. The general results for the propagation in arbitrary optical systems with finite-sized Gaussian apertures were also later derived ${ }^{5}$ within the framework of the ABCD matrix theorm ${ }^{10}$. However, this device will impact one of the two orthogonal field elements and thus random modulation is not introduced to the whole field. Its de-coherence ability is restricted to generating a partially coherent field. Another typical method is to implement the de-coherence and depolarization functionalities by a train of different optical devices. A polarized beam splitter is used to split the beam into two orthogonal polarized components, and then by moving phase diffusers e.g. ground glass plates, incoherent random phase retardation are introduced to one ${ }^{11}$, or both split fields ${ }^{6}$. Subsequently, these two beams are combined again to generate the polarization speckle. This is entirely feasible. However, it is understandable that this system might be too complex and too bulky for some applications. The other problem is the uncertainty associated with the system by these multi-individual devices.

SPECKLE 2015: VI International Conference on Speckle Metrology, edited by Fernando Mendoza Santoyo,

Eugenio R. Méndez, Proc. of SPIE Vol. 9660, 96601D · (c) 2015 SPIE · CCC code:

0277-786X/15/\$18 $\cdot$ doi: $10.1117 / 12.2189940$

Proc. of SPIE Vol. 9660 96601D-1 
The RRBS introduced in this paper, is a thin transmitting screen made of birefringent materials with random surface roughness. This thickness fluctuation will introduce random phase retardation in the incident field over the surface plane of RRBS and diffuse it like a traditional rough screen. At the same time, the birefringence of the material will cause the random phase difference proportional to local thickness between two orthogonal wave components and thus implement the random change of the polarization state for the field over the surface plane like a depolarizer. The comprehensive and rigorous analysis of this modulation behaviour is achieved in terms of the BCPM in the vector context. The relation between the modulation behaviour and the surface structure is revealed. Based on these BCPM expressions for the modulated field after depolarizers, the polarization speckle propagating through general optical systems including inherent Gaussian apertures are analysed with the Complex ABCD matrix, and the analytical results of the propagated BCPM are provided. Related statistical properties including the coherence and the polarization including their combined changes are also discussed. By these conclusions, it is found that the RRBS proposed here provides a flexible and easy method to implement random diffusion and depolarization to the incident field and the polarization speckle field with spatially varying polarization states is successfully generated. To provide the physical insight, numerical examples are cited and plotted in figures for this model. It is expected that the analytical result about the relation between the structural features of depolarizer and the statistical properties of the propagated polarization speckle patterns will not only facilitate the applications like retrieval method of birefringent depolarizer surface structure in speckle non-contact metrology, but also promote the design and optimization of modulation devices to generate the polarization speckle with prescribed statistical properties.

\section{FULLY DEVELOPED POLARIZAITON SPECKLE GENERATED BY RRBS}

The RRBS applied here is not a novel concept tracing back to a patent published in the US in $1969^{12}$. However, it is the first time to be considered for the special purpose to generate the polarization speckle field. Hence, its modulation is not yet analysed theoretically. In this paper, the research work will be carried out from the introduction on its structure and the related effect on the field modulation. As named, RRBS (figure1.(a)) is a thin transmitting screen made of birefringent materials with random surface roughness. It is placed in an optical system as illustrated in figure 1.(b).

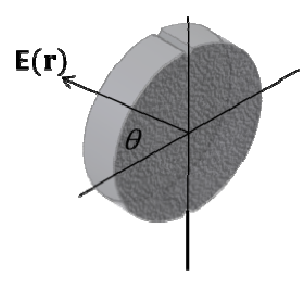

(a)

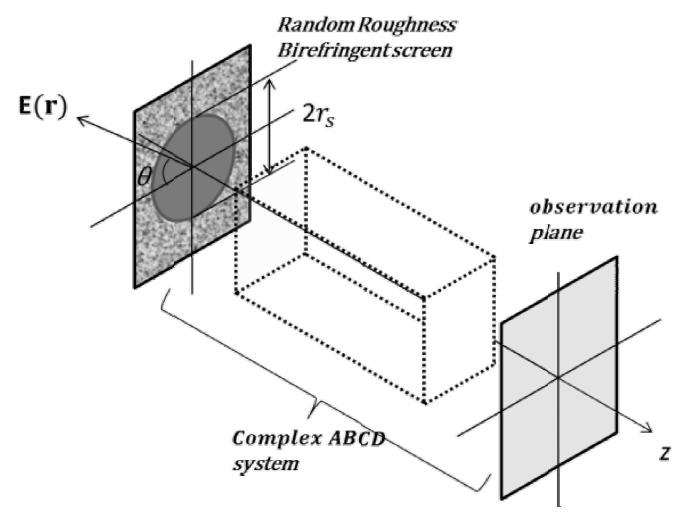

(b)

Figure 1. (a) RRBS and (b) the sketch of optical system including RRBS and complex ABCD propagation system

For a laser beam perpendicularly incident to the screen surface at point $\mathbf{r}$, the field vector $\mathbf{E}(\mathbf{r})=\left\{E_{x}(\mathbf{r}), E_{y}(\mathbf{r})\right\}$ is described with the components $E_{x}(\mathbf{r})$ and $E_{y}(\mathbf{r})$ polarized vertically and horizontally. Its $2 \times 2 \mathrm{BCPM}$ is defined as ${ }^{7}$

$$
\mathbf{W}\left(\mathbf{r}_{1}, \mathbf{r}_{2}\right)=\left\langle\mathbf{E}^{\dagger}\left(\mathbf{r}_{1}\right) \mathbf{E}\left(\mathbf{r}_{2}\right)\right\rangle=\left(\begin{array}{ll}
\left\langle E_{x}^{*}\left(\mathbf{r}_{1}\right) E_{x}\left(\mathbf{r}_{2}\right)\right\rangle & \left\langle E_{x}^{*}\left(\mathbf{r}_{1}\right) E_{y}\left(\mathbf{r}_{2}\right)\right\rangle \\
\left\langle E_{y}^{*}\left(\mathbf{r}_{1}\right) E_{x}\left(\mathbf{r}_{2}\right)\right\rangle & \left\langle E_{y}^{*}\left(\mathbf{r}_{1}\right) E_{y}\left(\mathbf{r}_{2}\right)\right\rangle
\end{array}\right),
$$

where the dagger $\square$ depicts the Hermitian conjugate, asterisk $\square$ means complex conjugate, and angular bracket $\langle\ldots\rangle$ denotes ensemble average. On the basis of this concept, the parameters of special interest: viz. the DoC $\eta$ and DoP P is determined by ${ }^{8,13}$ :

$$
\eta\left(\mathbf{r}_{1}, \mathbf{r}_{2}\right)=\operatorname{tr} \mathbf{W}\left(\mathbf{r}_{1}, \mathbf{r}_{2}\right)\left[\operatorname{tr} \mathbf{W}\left(\mathbf{r}_{1}, \mathbf{r}_{1}\right) \operatorname{tr} \mathbf{W}\left(\mathbf{r}_{2}, \mathbf{r}_{2}\right)\right]^{-1 / 2}
$$




$$
\mathrm{P}(\mathbf{r})=\left\{1-4 \operatorname{det} \mathbf{W}(\mathbf{r}, \mathbf{r})[\operatorname{tr} \mathbf{W}(\mathbf{r}, \mathbf{r})]^{-2}\right\}^{1 / 2},
$$

where $\operatorname{tr}$ and det mean the trace and determinant of the matrix, respectively. The BCPM is also easy to be converted into Stokes' parameters ${ }^{14}$ if found necessary.

For the field passing through a RRBS well aligned with the system, the vertical and horizontal polarized wave components are modulated by different phase retardations $\varphi_{m}(\mathbf{r}),(m=x, y)$, respectively. The emerging field $\mathbf{E}^{\prime}(\mathbf{r})=\mathbf{E}(\mathbf{r}) \mathbf{T}(\mathbf{r})$ is equal to the product of the incident vector $\mathbf{E}(\mathbf{r})$ with the local ray-transfer matrix (or so-called Jones matrix) $\mathbf{T}(\mathbf{r}){ }^{15,16}$ which is defined as

$$
\mathbf{T}(\mathbf{r})=\left(\begin{array}{cc}
e^{-j \varphi_{x}(\mathbf{r})} & 0 \\
0 & e^{-j \varphi_{y}(\mathbf{r})}
\end{array}\right)=\left(\begin{array}{cc}
e^{-j k n_{x} d(\mathbf{r})} & 0 \\
0 & e^{-j k n_{y} d(\mathbf{r})}
\end{array}\right),
$$

where $d(\mathbf{r})$ is the random local thickness, $k$ is the wavenumber, $j$ is the imaginary unite, while $n_{x}$ and $n_{y}=\varepsilon n_{x},(\varepsilon>1)$ denote the corresponding refractive indices. Therefore, a random phase difference $k n_{x}(\varepsilon-1) d(\mathbf{r})$ proportional to the random local thickness is introduced between two orthogonal wave components of $\mathbf{E}^{\prime}(\mathbf{r})$ and the random depolarization is introduced by this depolarizer. Now, the $\mathbf{W}^{\prime}\left(\mathbf{r}_{1}, \mathbf{r}_{2}\right)$ is given by,

$$
\mathbf{W}^{\prime}\left(\mathbf{r}_{1}, \mathbf{r}_{2}\right)=\left(\begin{array}{ll}
W_{x x}\left(\mathbf{r}_{1}, \mathbf{r}_{2}\right)\left\langle\exp \left\{j \Delta \varphi_{x x}\right\}\right\rangle & W_{x y}\left(\mathbf{r}_{1}, \mathbf{r}_{2}\right)\left\langle\exp \left\{j \Delta \varphi_{x y}\right\}\right\rangle \\
W_{y x}\left(\mathbf{r}_{1}, \mathbf{r}_{2}\right)\left\langle\exp \left\{j \Delta \varphi_{y x}\right\}\right\rangle & W_{y y}\left(\mathbf{r}_{1}, \mathbf{r}_{2}\right)\left\langle\exp \left\{j \Delta \varphi_{y y}\right\}\right\rangle
\end{array}\right)
$$

where $W_{m n},(m, n=x, y)$ is the matrix element of the $\mathbf{W}$ and $\Delta \varphi_{m n}=\varphi_{m}\left(\mathbf{r}_{1}\right)-\varphi_{n}\left(\mathbf{r}_{2}\right)$ is the phase differences totally depending on the structural features of the RRBS. Here, we assume the surface roughness is in accordance with the widely accepted Gaussian-Gaussian model in order to achieve analytical expressions ${ }^{17,18}$. Thus its thickness fluctuation is statistically defined in Gaussian terms with a zero mean and covariance $\sigma_{d}{ }^{2}$. Furthermore, with the hypothesis of widesense stationarity, the lateral correlation has a Gaussian dependency on the location distance $\Delta \mathbf{r}=\mathbf{r}_{1}-\mathbf{r}_{2}$ with a scale $r_{d}$, i.e.

$$
\left\langle d\left(\mathbf{r}_{1}\right) d\left(\mathbf{r}_{2}\right)\right\rangle=\sigma_{d}^{2} \exp \left\{-|\Delta \mathbf{r}|^{2} / r_{d}^{2}\right\}
$$

Since the $\Delta \varphi_{m n}$ is assumed a Gaussian process too, those ensemble average terms $\left\langle\Delta \varphi_{m n}\right\rangle$ should be the first-order characteristic function $^{18,19}$ of $\Delta \varphi_{m n}$ given by:

$$
\left\langle\Delta \varphi_{m n}\right\rangle=\exp \left\{\frac{k^{2} \sigma_{d}^{2}}{2}\left(\left(n_{m}-n_{n}\right)^{2}-\frac{2 n_{m} n_{n}|\Delta \mathbf{r}|^{2}}{r_{d}^{2}}\right)\right\}
$$

The double exponential function in the above equation will not render any analytical expressions for the propagated field. Depending on the different surface roughness, this expression could be approximated adequately. For a fully developed speckle generated by a heavy surface rough RRBS with large phase covariance $s^{2}=k^{2} \sigma_{d}{ }^{2} n_{x}{ }^{2}$, and small $r_{d}$, the measure of the lateral phase correlation length $r_{c}{ }^{2}=r_{d}{ }^{2} / s^{2}$ will be small. Therefore, the first two terms of the inner exponential functions' Taylor expansion are retained ${ }^{20,21}$ as its approximation. If the linearly polarized Gaussian incident beam has a polarization angle $\theta$ and $1 / e^{2}$ amplitude radius $r_{s}$, its $\operatorname{BCPM~} \mathbf{W}^{\prime}\left(\mathbf{r}_{1}, \mathbf{r}_{2}\right)$ is approximated in the form of:

$$
\mathbf{W}^{\prime}\left(\mathbf{r}_{1}, \mathbf{r}_{2}\right) \cong I_{o} \exp \left\{-\frac{\left|\mathbf{r}_{1}\right|^{2}+\left|\mathbf{r}_{2}\right|^{2}}{r_{s}^{2}}\right\}\left(\begin{array}{cc}
\cos ^{2} \theta \exp \left\{-|\Delta \mathbf{r}|^{2} / r_{c}^{2}\right\} & \cos \theta \sin \theta \exp \left\{-s^{2}(1-\varepsilon)^{2} / 2-\varepsilon|\Delta \mathbf{r}|^{2} / r_{c}^{2}\right\} \\
\cos \theta \sin \theta \exp \left\{-s^{2}(1-\varepsilon)^{2} / 2-\varepsilon|\Delta \mathbf{r}|^{2} / r_{c}^{2}\right\} & \sin ^{2} \theta \exp \left\{-\varepsilon^{2}|\Delta \mathbf{r}|^{2} / r_{c}^{2}\right\}
\end{array}\right)
$$

The corresponding DoP and DoC are given by 


$$
\begin{aligned}
& \mathrm{P}^{\prime}(\mathbf{r})=\left\{1-\sin ^{2} 2 \theta\left[1-\exp \left\{-s^{2}(1-\varepsilon)^{2}\right\}\right]\right\}^{1 / 2} ; \\
& \eta^{\prime}\left(\mathbf{r}_{1}, \mathbf{r}_{2}\right)=\cos ^{2} \theta \exp \left\{-|\Delta \mathbf{r}|^{2} / r_{c}^{2}\right\}+\sin ^{2} \theta \exp \left\{-\varepsilon^{2}|\Delta \mathbf{r}|^{2} / r_{c}^{2}\right\} .
\end{aligned}
$$

In this way, the relation between the structural features of RRBS and its simultaneous depolarization and de-coherence ability is revealed. The statistical properties of the propagated polarization speckle are sensitive to both the fine surface structure of this depolarizer introduced here and the following propagation system which will be discussed in the next section.

\section{PROPAGATION OF POLARIZAITON SPECKLE GENERATED BY RRBS}

Within the paraxial approximation, the field propagation within an optical system with inherent Gaussian apertures could be calculated within the framework of complex ABCD theory. The output field $\mathbf{E}^{o}(\mathbf{p})$ in the observation plane, is the integral of the modulated field $\mathbf{E}^{\prime}(\mathbf{r})$ and the Green's transform kernel $G(\mathbf{r}, \mathbf{p})$, which is given by ${ }^{10}$

$$
G(\mathbf{r}, \mathbf{p})=-\frac{j k}{2 \pi B} \exp \left\{-\frac{j k}{2 B}\left(A|\mathbf{r}|^{2}-2 \mathbf{r} \cdot \mathbf{p}+D|\mathbf{p}|^{2}\right)\right\} .
$$

Hence, the $\mathrm{BCPM} \mathbf{W}^{o}\left(\mathbf{p}_{1}, \mathbf{p}_{2}\right)$ is ${ }^{5}$

$$
\mathbf{W}^{o}\left(\mathbf{p}_{1}, \mathbf{p}_{2}\right)=\iint \mathbf{W}^{\prime}\left(\mathbf{r}_{1}, \mathbf{r}_{2}\right) G^{*}\left(\mathbf{r}_{1}, \mathbf{p}_{1}\right) G\left(\mathbf{r}_{2}, \mathbf{p}_{2}\right) d \mathbf{r}_{1} d \mathbf{r}_{2} .
$$

In the equations above, $A, B$ and $D$ are the complex elements of the $\mathrm{ABCD}$ matrix, which is determined by the multiplication of all individual optical components' ABCD matrices. Various typical optical components' matrices were provided in reference ${ }^{10}$, and the Gaussian shaped aperture was invoked for the sake of achieving analytical integrals and therefore provides attractable results of the general transmission function ${ }^{10}$. If we omitted the Gaussian beam size $r_{s}$ and properly compensated for this in the ABCD parameters, the general analytical results of the propagated field's BCPM $\mathbf{W}^{o}(\mathbf{p}, \mathbf{p})$ could be derived by the method introduced above:

$$
\mathbf{W}^{o}\left(\mathbf{p}_{1}, \mathbf{p}_{2}\right)=I_{o} \cos \theta \sin \theta\left(\begin{array}{cc}
\cot \theta W^{A B C D}\left(\mathbf{p}_{1}, \mathbf{p}_{2} ; r_{c}^{2}\right) & \exp \left\{-s^{2}(1-\varepsilon)^{2} / 2\right\} W^{A B C D}\left(\mathbf{p}_{1}, \mathbf{p}_{2} ; r_{c}^{2} / \varepsilon\right) \\
\exp \left\{-s^{2}(1-\varepsilon)^{2} / 2\right\} W^{A B C D}\left(\mathbf{p}_{1}, \mathbf{p}_{2} ; r_{c}^{2} / \varepsilon\right) & \tan \theta W^{A B C D}\left(\mathbf{p}_{1}, \mathbf{p}_{2} ; r_{c}^{2} / \varepsilon^{2}\right)
\end{array}\right)
$$

$W^{A B C D}\left(\mathbf{p}_{1}, \mathbf{p}_{2} ; \mu^{2}\right)$ with parameter $\mu$ is a function defined as

$$
\begin{gathered}
W^{A B C D}\left(\mathbf{p}_{1}, \mathbf{p}_{2} ; \mu^{2}\right)=\frac{\mu^{2}}{4 \operatorname{Im}\left[A^{*} B\right] / k+\mu^{2}|A|^{2}} \exp \left\{\frac{-j\left(\left|\mathbf{p}_{1}\right|^{2}-\left|\mathbf{p}_{2}\right|^{2}\right)\left\{\operatorname{Im}\left[B^{2}\right]-2 \operatorname{Im}\left[A^{*} B\right] \operatorname{Re}\left[D^{*} B\right]+\frac{k \mu^{2}}{2}\left(\operatorname{Re}[A B]-\operatorname{Re}\left[D^{*} B\right]|A|^{2}\right)\right\}}{\left(4 \operatorname{Im}\left[A^{*} B\right] / k+\mu^{2}|A|^{2}\right)|B|^{2}}\right\} \\
\exp \left\{\frac{-\left(\mathbf{p}_{1}-\mathbf{p}_{2}\right)^{2}}{4 \operatorname{Im}\left[A^{*} B\right] / k+\mu^{2}|A|^{2}}\right\} \exp \left\{\frac{-\left(\left|\mathbf{p}_{1}\right|^{2}+\left|\mathbf{p}_{2}\right|^{2}\right)\left\{-2 \operatorname{Im}[B]^{2}+2 \operatorname{Im}\left[A^{*} B\right] \operatorname{Im}\left[D^{*} B\right]+\frac{k \mu^{2}}{2}\left(|A|^{2} \operatorname{Im}\left[B^{*} D\right]-\operatorname{Im}[A B]\right)\right\}}{\left(4 \operatorname{Im}\left[A^{*} B\right] / k+\mu^{2}|A|^{2}\right)|B|^{2}}\right\}
\end{gathered}
$$

Equation (12) is the primary conclusion for the propagated polarization speckle generated by the RRBS. Based on these analytical results, the corresponding DOC and DOP for different propagation systems can be calculated easily in order to study the statistical properties of the polarization speckle's coherence and polarization states.

A free space system with a propagation distance $z$ gives an ABCD matrix having the elements $A=1-j z / z_{R}, B=z$, and $D=1$, where $z_{R}=k r_{s}{ }^{2} / 2$ is the Rayleigh range. The $r_{s}=1 \mathrm{~mm}, r_{c}=0.1 \mathrm{~mm}$, wavelength $\lambda=0.633 \mu \mathrm{m}$, and $s^{2}=100$ are taken to represent a numerical demonstration. For the DoP $P_{\text {free space }}(\mathbf{p})$ of the speckle on the observation screen, the variation with the normalized propagation distance $z / z_{R}$ and the normalized location $\mathbf{p} / r_{s}$ in the observation plane is 
shown in figure 2. From these plots, the non-uniform distribution of the polarization on the beam cross section and its variation in propagation are demonstrated. For material with stronger birefringence, more significant DoP spatial variation will be observed. At the same time, the $\operatorname{DoC} \eta_{\text {free space }}(\mathbf{p},-\mathbf{p})$ for two symmetrical points $\mathbf{p}$ and $-\mathbf{p}$ with respect to the normalized propagation distance $z / z_{R}$ and normalized $\mathbf{p} / r_{s}$ is illustrated in figure 3. The DoC increases during propagation to the far field and approaches unity, which is in accordance with the Van Cittert-Zernike theorem. The sensitivity of the $\mathrm{DoC}$ and the DoP to the polarization angle of the incident field is also indicated by these figures.

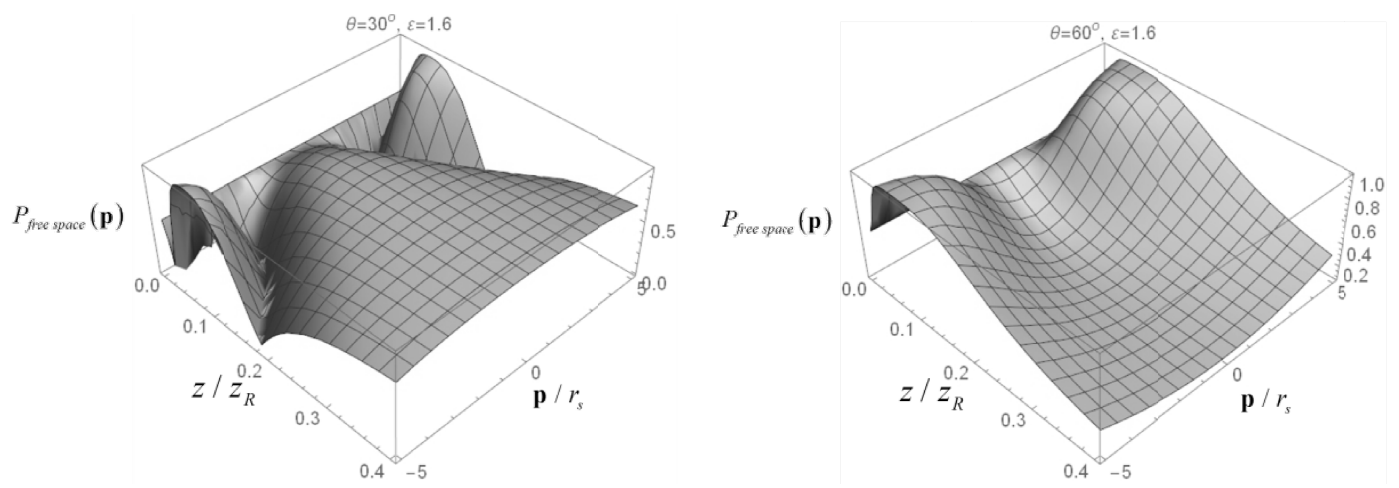

Figure 2. $P_{\text {free space }}(\mathbf{p})$ of the fully developed polarization speckle for a free space system for selected polarization angles

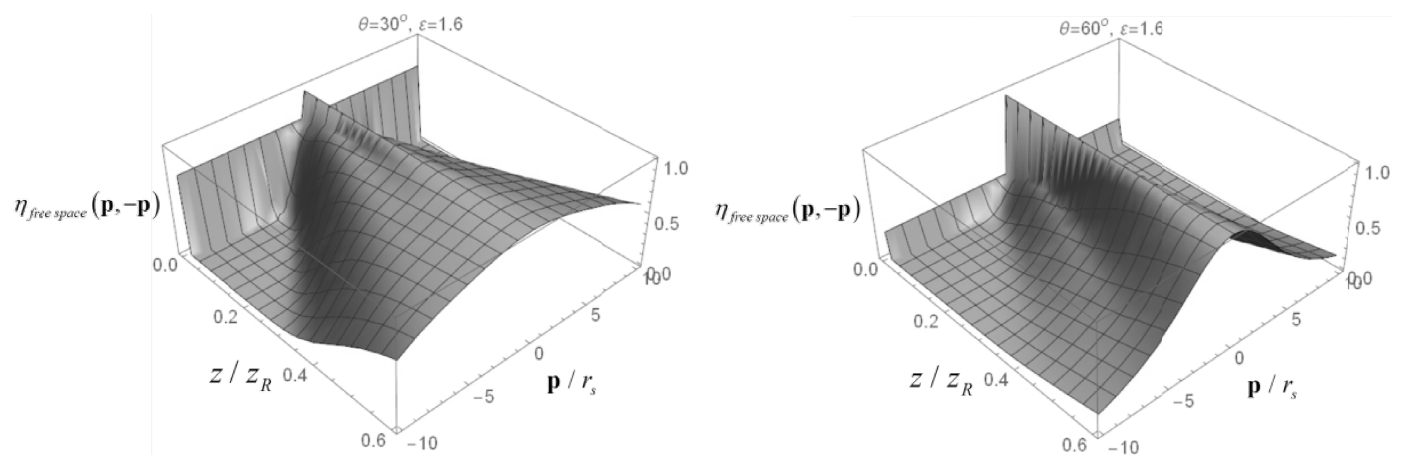

Figure 3. $\eta_{\text {freespace }}(\mathbf{p},-\mathbf{p})$ of the fully developed polarization speckle for a free space system for the selected polarization angles

\section{DISUCSSION AND CONCLUSION}

In this paper, we have proposed a generating method of polarization speckle with spatial variation of the polarization state. The ability of RRBS to simultaneously diffuse and depolarize as a compact single-device system is approved by the analytical results of corresponding BCPM of the generated field with the statistical parameters DoC and DoP. The changes of the coherence and polarization state during propagation are studied within the framework of ABCD theory for general optical systems, and specifically a free space system is cited as example to provide physical insight. Though we only discussed the fully developed polarization speckle generated by a RRBS with large phase phase covariance and small lateral correlation length here, other kinds of polarization speckles like the partially developed ones generated by gentle roughness models of RRBS could also be analysed in similar steps and the conclusions will be presented in our future papers. This rigorous analysis for the RRBS might help researchers in the development of the polarization speckle generating method and facilitate the improvement of speckle non-contact metrology for birefringent rough objects. 


\section{REFERENCES}

[1] Goodman, J. W., [Speckle phenomena in optics: theory and applications], Roberts \& Company Publishers, (2007).

[2] James, D., "Change of polarization of light beams on propagation in free space," J. Opt. Soc. Am. A 11(5), 1641 (1994).

[3] Agrawal, G. P., Wolf, E., "Propagation-induced polarization changes in partially coherent optical beams," J. Opt. Soc. Am. A 17(11), 2019 (2000).

[4] Shirai, T., Wolf, E., "Coherence and polarization of electromagnetic beams modulated by random phase screens and their changes on propagation in free space," J. Opt. Soc. Am. A 21(10), 1907-1916 (2004).

[5] Hanson, S. G., Wang, W., Jakobsen, M. L., Takeda, M., "Coherence and polarization of electromagnetic beams modulated by random phase screens and their changes through complex ABCD optical systems," J. Opt. Soc. Am. A 25(9), 2338-2346 (2008).

[6] Takeda, M., Wang, W., Hanson, S. G., "Polarization speckles and generalized Stokes vector wave: a review," Proc. SPIE 7387, V - 1-V - 7 (2010).

[7] Gori, F., Santarsiero, M., Vicalvi, S., Borghi, R., Guattari, G., "Beam coherence-polarization matrix," Pure Appl. Opt. J. Eur. Opt. Soc. Part A 7, 941 (1998).

[8] Wolf, E., "Unified theory of coherence and polarization of random electromagnetic beams," Phys. Lett. A 312, 263-267 (2003).

[9] Scudieri, F., Bertolotti, M., Bartolino, R., "Light scattered by a liquid crystal: a new quasi-thermal source," Appl. Opt. 13, 181-185 (1974).

[10] Yura, H., Hanson, S. G., "Optical beam wave propagation through complex optical systems," J. Opt. Soc. Am. A 4(10), 1931-1948 (1987).

[11] Kumar Singh, R., Naik, D. N., Itou, H., Miyamoto, Y., Takeda, M., Singh, R. K., Naik, D. N., Itou, H., Miyamoto, Y., "Characterization of spatial polarization fluctuations in scattered field," J. Opt. 105010(10), (2014).

[12] Peters, C. J., "Light depolarizer," US3433553 A, USA (1969).

[13] [13] Wolf, E., [Introduction to the Theory of Coherence and Polarization of Light], Cambridge University Press, (2007).

[14] Wolf, E., "Polarization invariance in beam propagation," Opt. Lett. 32(23), 3400 (2007).

[15] Mandel, L., Wolf, E., [Optical Coherence and Quantum Optics], Cambridge University Press, (1995).

[16] Goodman, J. W., [Introduction to Fourier Optics], McGraw-Hill 35 (1988).

[17] Dainty, J. C., Ennos, A. E., Franqo, M., Goodman, J. W., McKechnie, T. S., Parry, G., [Laser Speckle and related phenomena], Springer-Verlag Berlin and New York (1975).

[18] Goodman, J. W., [statistical optics], Wiley-Interscience, New York (2000).

[19] Wasserman, L., [All of Statistics: A Concise Course in Statistical Inference], Springer Science \& Business Media (2004).

[20] Hyde, M. W., Basu, S., Spencer, M. F., Cusumano, S. J., Fiorino, S. T., "Physical optics solution for the scattering of a partially-coherent wave from a statistically rough material surface", Opt. Express 21(6), 6807-6825 (2013).

[21] Rose, B., Imam, H., Hanson, S. G.., Yura, H. T., "Effects of target structure on the performance of laser time-offlight velocimeter systems," Appl. Opt. 36(2), 518-533 (1997). 